\title{
Analysis of the Convergence and Periodicity of a Rational Difference Equation
}

\author{
Mohammed B. Almatrafi ${ }^{1 *}$ and Marwa M. Alzubaidi ${ }^{2}$ \\ ${ }^{1}$ Department of Mathematics, College of Science, Taibah University, Al-Madinah Al-Munawarah, Saudi Arabia \\ ${ }^{2}$ Department of Mathematics, College of Duba, University of Tabuk, Saudia Arabia \\ *Corresponding author E-mail: mmutrafi@taibahu.edu.sa
}

\author{
Article Info \\ Keywords: Difference equation, Equi- \\ libria, Global attractivity, Local stabil- \\ ity, Periodicity \\ 2010 AMS: 39 A10 \\ Received: 02 May 2019 \\ Accepted: 28 July 2019 \\ Available online: 26 December 2019
}

\begin{abstract}
The exact solutions of most difference equations cannot be obtained sometimes. This can be attributed to the fact that there is no a specific approach from which one can find the exact solution. Therefore, many researchers tend to study the qualitative behaviours of these equations. In this paper, we will investigate some qualitative properties such as local stability, global stability, periodicity and solutions of the following eighth order recursive equation
\end{abstract}

$$
x_{n+1}=c_{1} x_{n-3}-\frac{c_{2} x_{n-3}}{c_{3} x_{n-3}-c_{4} x_{n-7}}, \quad n=0,1, \ldots,
$$

where the coefficients $c_{i}$, for all $i=1, \ldots, 4$, are assumed to be positive real numbers and the initial conditions $x_{i}$ for all $i=-7,-6, \ldots, 0$, are arbitrary non-zero real numbers.

\section{Introduction}

Nowadays, a huge number of researchers put a lot of effort to investigate the qualitative behaviours of some fractional recursive equations Researchers examine some properties such as local stability, global stability, boundedness, periodicity and theoretical and numerical solutions to predict the future pattern of these equations. This development can be obviously seen in most recent studies. Take, for instance the following ones. Almatrafi et al. [1] discovered the stability, periodicity, boundedness and solutions of the following fourth order fractional difference equations

$$
x_{n+1}=\frac{\alpha x_{n} x_{n-3}}{ \pm \beta x_{n-3} \pm \gamma x_{n-2}} .
$$

Cinar [2] obtained the solution of the second order recursive equation

$$
x_{n+1}=\frac{a x_{n-1}}{1+b x_{n} x_{n-1}} .
$$

Elabbasy et al. [3] examined the qualitative behaviours of the recursive equation

$$
x_{n+1}=a x_{n}-\frac{b x_{n}}{c x_{n}-d x_{n-1}} .
$$

Garić-Demirović et al. [4] investigated the periodicity of the solution and the stability of the equilibrium point of the difference equation

$$
x_{n+1}=\frac{A x_{n}^{2}+B x_{n} x_{n-1}+C x_{n-1}^{2}}{a x_{n}^{2}+b x_{n} x_{n-1}+c x_{n-1}^{2}} .
$$

Email addresses and ORCID numbers: mmutrafi@taibahu.edu.sa, https://orcid.org/0000-0002-6859-2028 (M. B. Almatrafi), mmialzubaidi@hotmail.com, https://orcid.org/0000-0002-3314-5244 (M. M. Alzubaidi) 
In [5], the authors concerned with presenting the qualitative behaviour of the sixth order difference equation

$$
x_{n+1}=\frac{C x_{n-5}}{A+B x_{n-2} x_{n-5}} .
$$

Khyat et al. [6] analysed the properties of the following second order recursive equation

$$
x_{n+1}=\frac{x_{n}}{C x_{n-1}^{2}+D x_{n}+F} .
$$

The investigation in [7] concentrates on showing the periodic character, semi-cycle character and global stability of the difference equation

$$
x_{n+1}=\frac{\alpha+\beta x_{n}+\gamma x_{n-k}}{B x_{n}+C x_{n-k}} .
$$

Simsek et al [8] obtained the expressions of the solutions of the fourth order difference equation

$$
x_{n+1}=\frac{x_{n-3}}{1+x_{n-1}}
$$

More results on the qualitative behaviours of some fractional difference equations can be obtained on refs. [9]-[19].

Our principal aim in this work is to discuss some mathematical properties such as local stability, global attractivity, periodic character and solutions of the eighth order difference equation

$$
x_{n+1}=c_{1} x_{n-3}-\frac{c_{2} x_{n-3}}{c_{3} x_{n-3}-c_{4} x_{n-7}}, \quad n=0,1, \ldots
$$

where the coefficients $c_{i}$, for all $i=1, \ldots, 4$, are assumed to be positive real numbers and the initial conditions are required to be arbitrarily real numbers. Moreover, theoretical and numerical solutions to a special case of Eq.(1.1) will be shown in this paper.

\section{Local stability of the equilibrium point}

The main duty in this section is to analyse the behaviour of the solutions in the neighbourhood of the equilibrium point. The equilibrium point of Eq.(1.1) is given by

$$
\bar{x}=c_{1} \bar{x}-\frac{c_{2} \bar{x}}{c_{3} \bar{x}-c_{4} \bar{x}}
$$

Hence,

$$
\bar{x}=\frac{c_{2}}{\left(1-c_{1}\right)\left(c_{4}-c_{3}\right)}, c_{1} \neq 1, c_{3} \neq c_{4}
$$

Next, we assume that a function $h:(0, \infty)^{2} \longrightarrow(0, \infty)$ is defined by the form

$$
h(y, z)=c_{1} y-\frac{c_{2} y}{c_{3} y-c_{4} z} .
$$

Then,

$$
\begin{aligned}
\frac{\partial h(y, z)}{\partial y} & =c_{1}-\frac{c_{2}\left(c_{3} y-c_{4} z\right)-c_{2} c_{3} y}{\left(c_{3} y-c_{4} z\right)^{2}}=c_{1}+\frac{c_{2} c_{4} z}{\left(c_{3} y-c_{4} z\right)^{2}} \\
\frac{\partial h(y, z)}{\partial z} & =-\frac{c_{2} c_{4} y}{\left(c_{3} y-c_{4} z\right)^{2}} .
\end{aligned}
$$

We now calculate Eq.(2.2) and Eq.(2.3) at $\bar{x}$ as follows:

$$
\begin{aligned}
\frac{\partial h(\bar{x}, \bar{x})}{\partial y} & =c_{1}+\frac{c_{2} c_{4} \bar{x}}{\left(c_{3} \bar{x}-c_{4} \bar{x}\right)^{2}}=c_{1}+\frac{c_{4}\left(1-c_{1}\right)}{c_{4}-c_{3}}:=-p_{1}, \\
\frac{\partial h(\bar{x}, \bar{x})}{\partial z} & =-\frac{c_{2} c_{4} \bar{x}}{\left(c_{3} \bar{x}-c_{4} \bar{x}\right)^{2}}=-\frac{c_{4}\left(1-c_{1}\right)}{c_{4}-c_{3}}:=-p_{2} .
\end{aligned}
$$

Thus, the linearised equation of Eq. (1.1) around $\bar{x}$ is given by the form:

$$
u_{n+1}+p_{1} u_{n-3}+p_{2} u_{n-7}=0
$$

Theorem 2.1. Let

$$
\left|c_{4}-c_{1} c_{3}\right|+c_{4}\left|1-c_{1}\right|<\left|c_{4}-c_{3}\right| .
$$

Then, the equilibrium point of Eq.(1.1) is locally asymptotically stable. 
Proof. Theorem A in [12] guarantees that the equilibrium point of Eq.(1.1) is locally asymptotically stable if

$$
\left|p_{1}\right|+\left|p_{2}\right|<1,
$$

which leads to

$$
\left|-\left(c_{1}+\frac{c_{4}\left(1-c_{1}\right)}{c_{4}-c_{3}}\right)\right|+\left|\frac{c_{4}\left(1-c_{1}\right)}{c_{4}-c_{3}}\right|<1 .
$$

Therefore,

$$
\left|c_{1}\left(c_{4}-c_{3}\right)+c_{4}\left(1-c_{1}\right)\right|+c_{4}\left|1-c_{1}\right|<\left|c_{4}-c_{3}\right| .
$$

Or,

$$
\left|c_{4}-c_{1} c_{3}\right|+c_{4}\left|1-c_{1}\right|<\left|c_{4}-c_{3}\right|
$$

The proof is complete.

\section{Global stability of the equilibrium point}

In this section, we will present a specific condition under which the equilibrium point is a global stable.

Theorem 3.1. The equilibrium point of Eq.(1.1) is a global attractor if $c_{1}<1$.

Proof. Assume that $a, b \in \mathbb{R}$ and let $h:[a, b]^{2} \longrightarrow[a, b]$ be a function defined by Eq.(2.1). Then, the function $h$ is increasing in $y$ and decreasing in $z$. Next, we suppose that $(\phi, \psi)$ is a solution to the following system:

$$
\phi=h(\phi, \psi), \psi=h(\psi, \phi)
$$

Thus,

$$
\begin{aligned}
& \phi \quad=h(\phi, \psi)=c_{1} \phi-\frac{c_{2} \phi}{c_{3} \phi-c_{4} \psi} \\
& \psi \quad=h(\psi, \phi)=c_{1} \psi-\frac{c_{2} \psi}{c_{3} \psi-c_{4} \phi} .
\end{aligned}
$$

Simplifying this gives us

$$
\begin{aligned}
c_{3} \phi^{2}-c_{4} \phi \psi & =c_{1} c_{3} \phi^{2}-c_{1} c_{4} \phi \psi-c_{2} \phi \\
c_{3} \psi^{2}-c_{4} \phi \psi & =c_{1} c_{3} \psi^{2}-c_{1} c_{4} \phi \psi-c_{2} \psi
\end{aligned}
$$

Subtracting Eq.(3.2) from Eq.(3.1) yields

$$
c_{3}\left(\phi^{2}-\psi^{2}\right)=c_{1} c_{3}\left(\phi^{2}-\psi^{2}\right)+c_{2}(\psi-\phi)
$$

Therefore,

$$
(\phi-\psi)\left[c_{3}\left(1-c_{1}\right)(\phi+\psi)+c_{2}\right]=0 .
$$

Hence, if $c_{1}<1$, then $\phi=\psi$. As a result, Theorem B in [20] assures that the equilibrium point is a global attractor.

\section{Periodicity of the solutions}

This section is devoted to study the periodicity of the solution of Eq.(1.1).

Theorem 4.1. Eq.(1.1) has no prime period two solutions.

Proof. Suppose that Eq.(1.1) has prime period two solutions on the form:

$$
\ldots, t, \tau, t, \tau, \ldots
$$

where $t \neq \tau$. Then, Eq.(1.1) leads to

$$
\begin{aligned}
t & =c_{1} t-\frac{c_{2} t}{c_{3} t-c_{4} t}, \\
\tau & =c_{1} \tau-\frac{c_{2} \tau}{c_{3} \tau-c_{4} \tau} .
\end{aligned}
$$

Therefore,

$$
\begin{aligned}
\left(1-c_{1}\right) t & =-\frac{c_{2}}{c_{3}-c_{4}}, \\
\left(1-c_{1}\right) \tau & =-\frac{c_{2}}{c_{3}-c_{4}} .
\end{aligned}
$$

This exactly implies that $t=\tau$, which contradicts our assumption. 


\section{Special case of Eq.(1.1)}

We now turn to solve the following difference equation theoretically.

$$
x_{n+1}=x_{n-3}-\frac{x_{n-3}}{x_{n-3}-x_{n-7}}, \quad n=0,1, \ldots
$$

Theorem 5.1. Let $\left\{x_{n}\right\}_{n=-7}^{\infty}$ be a solution to Eq.(5.1) and assume that $x_{-7}=\alpha, x_{-6}=\beta, x_{-5}=\gamma, x_{-4}=\delta, x_{-3}=\kappa, x_{-2}=\lambda, x_{-1}=$ $\mu, x_{0}=\rho$. Then, for $n=0,1,2, \ldots$, the solution of Eq.(5.1) is given by the following formulas:

$$
\begin{aligned}
& x_{8 n-7}=-\frac{[(n-1) \alpha-n \kappa][\alpha-\kappa+n]}{\alpha-\kappa}, \quad x_{8 n-6}=-\frac{[(n-1) \beta-n \lambda][\beta-\lambda+n]}{\beta-\lambda}, \\
& x_{8 n-5}=-\frac{[(n-1) \gamma-n \mu][\gamma-\mu+n]}{\gamma-\mu}, \quad x_{8 n-4}=-\frac{[(n-1) \delta-n \rho][\delta-\rho+n]}{\delta-\rho}, \\
& x_{8 n-3}=-\frac{[n \alpha-(n+1) \kappa][\alpha-\kappa+n]}{\alpha-\kappa}, \quad x_{8 n-2}=-\frac{[n \beta-(n+1) \lambda][\beta-\lambda+n]}{\beta-\lambda}, \\
& x_{8 n-1}=-\frac{[n \gamma-(n+1) \mu][\gamma-\mu+n]}{\gamma-\mu}, \quad x_{8 n}=-\frac{[n \delta-(n+1) \rho][\delta-\rho+n]}{\delta-\rho} .
\end{aligned}
$$

Proof. It can be easily seen that the solution is true at $n=0$. Now, we suppose that $n>0$ and assume that the relations are satisfied at $n-1$ as follows:

$$
\begin{aligned}
& x_{8 n-15}=-\frac{[(n-2) \alpha-(n-1) \kappa][\alpha-\kappa+n-1]}{\alpha-\kappa}, x_{8 n-14}=-\frac{[(n-2) \beta-(n-1) \lambda][\beta-\lambda+n-1]}{\beta-\lambda}, \\
& x_{8 n-13}=-\frac{[(n-2) \gamma-(n-1) \mu][\gamma-\mu+n-1]}{\gamma-\mu}, x_{8 n-12}=-\frac{[(n-2) \delta-(n-1) \rho][\delta-\rho+n-1]}{\delta-\rho}, \\
& x_{8 n-11}=-\frac{[(n-1) \alpha-n \kappa][\alpha-\kappa+n-1]}{\alpha-\kappa}, \quad x_{8 n-10}=-\frac{[(n-1) \beta-n \lambda][\beta-\lambda+n-1]}{\beta-\lambda}, \\
& x_{8 n-9}=-\frac{[(n-1) \gamma-n \mu][\gamma-\mu+n-1]}{\gamma-\mu}, \quad x_{8 n-8}=-\frac{[(n-1) \delta-n \rho][\delta-\rho+n-1]}{\delta-\rho} .
\end{aligned}
$$

Next, it can be obviously observed from Eq.(5.1) that

$$
\begin{aligned}
x_{8 n-7} & =x_{8 n-11}-\frac{x_{8 n-11}}{x_{8 n-11}-x_{8 n-15}} \\
& =-\frac{[(n-1) \alpha-n \kappa][\alpha-\kappa+n-1]}{\alpha-\kappa}-\frac{-\frac{[(n-1) \alpha-n \kappa][\alpha-\kappa+n-1]}{\alpha-\kappa}}{-\frac{[(n-1) \alpha-n \kappa][\alpha-\kappa+n-1]}{\alpha-\kappa}+\frac{[(n-2) \alpha-(n-1) \kappa][\alpha-\kappa+n-1]}{\alpha-\kappa}} \\
& =-\frac{[(n-1) \alpha-n \kappa][\alpha-\kappa+n-1]}{\alpha-\kappa}+\frac{[(n-1) \alpha-n \kappa]}{\kappa-\alpha} \\
& =-\frac{[(n-1) \alpha-n \kappa][\alpha-\kappa+n]}{\alpha-\kappa} . \\
x_{8 n-6} & x_{8 n-10-\frac{x_{8 n-10}}{x_{8 n-10-x_{8 n-14}}}} \\
& =-\frac{[(n-1) \beta-n \lambda][\beta-\lambda+n-1]}{\beta-\lambda}-\frac{-\frac{[(n-1) \beta-n \lambda][\beta-\lambda+n-1]}{\beta-\lambda}}{-\frac{[(n-1) \beta-n \lambda][\beta-\lambda+n-1]}{\beta-\lambda}+\frac{[(n-2) \beta-(n-1) \lambda][\beta-\lambda+n-1]}{\beta-\lambda}} \\
& =-\frac{[(n-1) \beta-n \lambda][\beta-\lambda+n-1]}{\beta-\lambda}+\frac{[(n-1) \beta-n \lambda]}{\lambda-\beta} \\
& =-\frac{[(n-1) \beta-n \lambda][\beta-\lambda+n]}{\beta-\lambda} .
\end{aligned}
$$

Other formulas can be proved in a similar way. Thus, the remaining proofs will be omitted.

\section{Numerical examples}

In order to confirm our theoretical work, we will illustrate some figures that show the behaviour of the solutions according to the previous conditions. 
Example 6.1. The local stability of the equilibrium point is depicted in this example under the values $c_{1}=0.3, c_{2}=0.1, c_{3}=8, c_{4}=$ $1, x_{-7}=0.001, x_{-6}=-0.02, x_{-5}=-0.03 . x_{-4}=0.02 . x_{-3}=-0.04 . x_{-7}=0.021 . x_{-1}=-0.01 . x_{0}=-0.02$. See Figure 6.1.

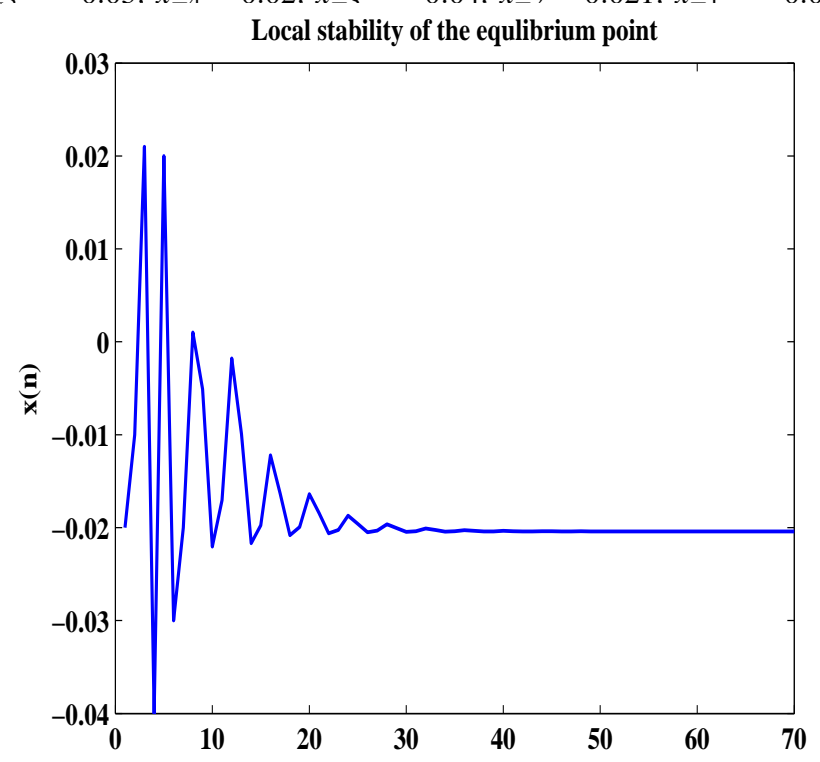

Figure 6.1: Local Stability of The Equilibrium Point.

Example 6.2. The global stability of the equilibrium point is given in Figure 6.2 according to the following data. $c_{1}=0.4, c_{2}=0.2, c_{3}=$ $8, c_{4}=1, x_{-7}=0.1, x_{-6}=-0.2, x_{-5}=6, x_{-4}=-5, x_{-3}=3, x_{-2}=-1, x_{-1}=1, x_{0}=-0.2$.

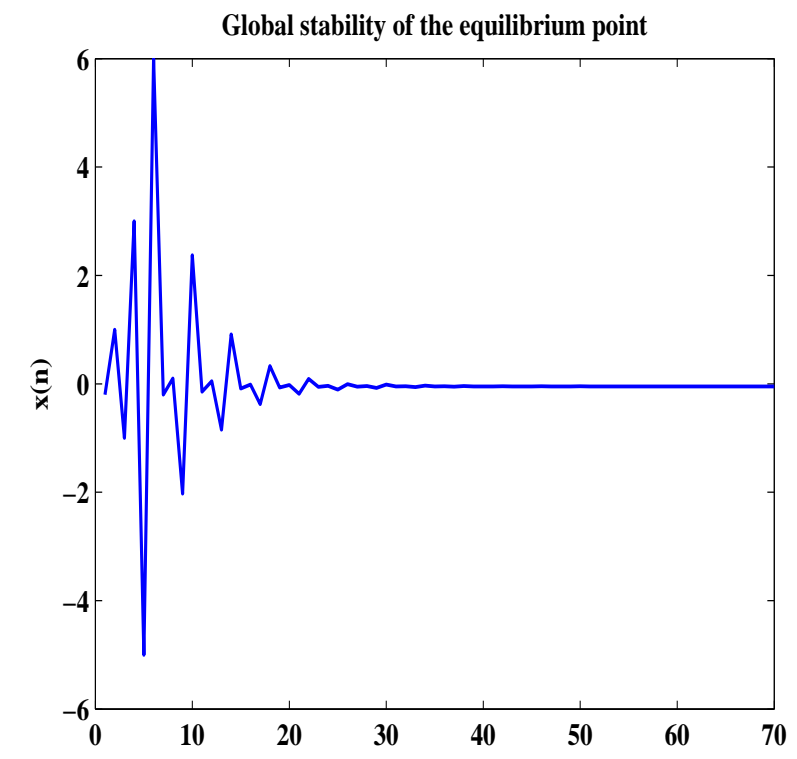

Figure 6.2: Global Stability of The Equilibrium Point. 
Example 6.3. In Figure 6.3, we plot another behaviour of the solutions of Eq.(1.1). Here, we assume that $c_{1}=0.6, c_{2}=0.2, c_{3}=4, c_{4}=$ $0.2, x_{-7}=-1, x_{-6}=-0.2, x_{-5}=0.2, x_{-4}=1, x_{-3}=0.1, x_{-2}=-0.5, x_{-1}=0.25, x_{0}=-0.3$.

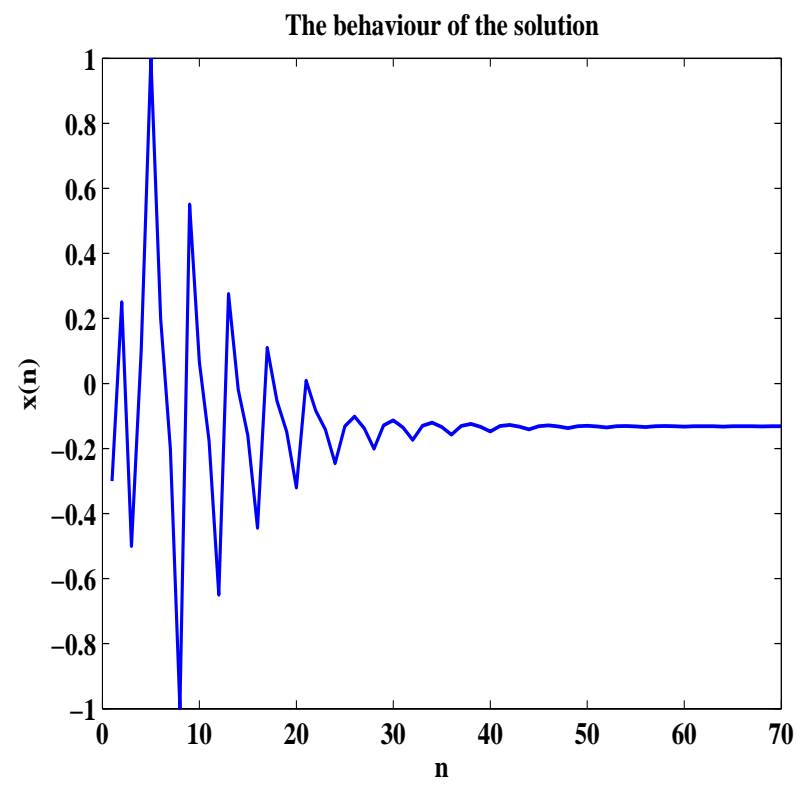

Figure 6.3: Solution of Eq.(1.1).

Example 6.4. Figure 6.4 shows the solution of the special case equation when we take $x_{-7}=-0.8, x_{-6}=0.2, x_{-5}=0.7, x_{-4}=1.5, x_{-3}=$ $-0.1, x_{-2}=0.5, x_{-1}=0.12, x_{0}=-1$.

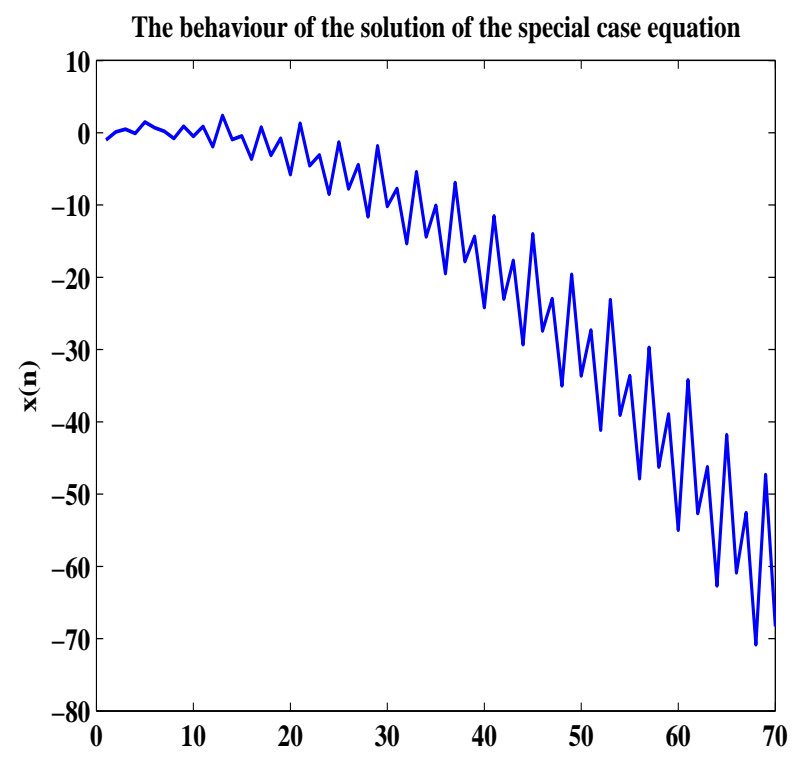

Figure 6.4: Solution of the Special Case Equation.

\section{Conclusion}

In this work, we have explored the stability and periodicity of Eq.(1.1) and analysed the solutions of Eq.(5.1). Section 2 highlighted a condition under which the equilibrium point of Eq.(1.1) is locally asymptotically stable. Following this, we have shown that the equilibrium point is a global stable if $c_{1}<1$, as pictured in Figure 6.2. In Section 4, it has been proved that Eq.(1.1) has no prime period two solutions. Finally, the analytical and numerical solutions of Eq.(5.1) has been provided in Theorem 5.1 and Section 6, respectively. 


\section{References}

[1] M. B. Almatrafi, E. M. Elsayed, F. Alzahrani, Qualitative behavior of two rational difference equations, Fundam. J. Math. Appl., 1(2) (2018), 194-204

[2] C. Cinar, On the positive solutions of the difference equation $x_{n+1}=a x_{n-1} /\left(1+b x_{n} x_{n-1}\right)$, Appl. Math. Comput., 156 (2004), $587-590$.

[3] E. M. Elabbasy, H. El-Metwally, E. M. Elsayed, On the difference equation $x_{n+1}=a x_{n}-\left(b x_{n}\right) /\left(c x_{n}-d x_{n-1}\right)$, Adv. Difference Equ., 2006 (2006), Article ID $82579,1-10$.

[4] M. Garić-Demirović, M. Nurkanović, Z. Nurkanović, Stability, periodicity and Neimark-Sacker bifurcation of certain homogeneous fractional difference equations, Int. J. Difference Equ., 12(1) (2017), 27-53.

[5] M. Ghazel, E.M. Elsayed, A.E. Matouk, A.M. Mousallam, Investigating dynamical behaviors of the difference equation $x_{n+1}=C x_{n-5} /\left(A+B x_{n-2} x_{n-5}\right)$, J. Nonlinear Sci. Appl., 10 (2017), 4662-4679.

[6] T. Khyat, M. R. S. Kulenović, The invariant curve caused by Neimark-Sacker bifurcation of a perturbed Beverton-Holt difference equation, Int. J. Difference Equ., 12(2) (2017), 267-280.

[7] M. Saleh, N. Alkoumi, Aseel Farhat, On the dynamic of a rational difference equation $x_{n+1}=\alpha+\beta x_{n}+\gamma x_{n-k} / B x_{n}+C x_{n-k}$, Chaos, Solitons Fractals, 96(2017), 76-84

[8] D. Simsek, C. Cinar, I. Yalcinkaya, On the recursive sequence $x_{n+1}=\frac{x_{n-3}}{1+x_{n-1}}$, Int. J. Contemp. Math. Sci., 1(10) (2006), 475-480.

[9] M. B. Almatrafi, E. M. Elsayed, Solutions and formulae for some systems of difference equations, MathLAB J., 1(3) (2018), 356-369.

[10] M. B. Almatrafi, E. M. Elsayed, Faris Alzahrani, Qualitative behavior of a quadratic second-order rational difference equation, Int. J. Adv. Math., 2019(1) (2019), 1-14.

[11] F. Belhannache, N. Touafek, R. Abo-zeid, On a higher-order rational difference equation, J. Appl. Math. \& Informatics, 34(5-6) (2016), 369-382.

[12] E. M. Elabbasy, H. El-Metawally, E. M. Elsayed, On the difference equation $x_{n+1}=\left(a x_{n}^{2}+b x_{n-1} x_{n-k}\right) /\left(c x_{n}^{2}+d x_{n-1} x_{n-k}\right)$, Sarajevo J. Math., 4(17) (2008), 1-10.

[13] M. A. El-Moneam, E.M.E. Zayed, Dynamics of the rational difference equation, Inform. Sci. Letters, 3(2) (2014), 45-53.

[14] A. Khaliq, Sk.S. Hassan, Dynamics of a rational difference equation $x_{n+1}=a x_{n}+\left(\alpha+\beta x_{n-k}\right) /\left(A+B x_{n-k}\right)$, Int. J. Adv. Math., 2018(1) (2018), $159-179$.

[15] V. L. Kocic, G. Ladas, Global Behaviour of Nonlinear Difference Equations of Higher Order with Applications, Kluwer Academic Publishers, Dordrecht, 1993.

[16] Y. Kostrov, Z. Kudlak, On a second-order rational difference equation with a quadratic term, Int. J. Difference Equ., 11(2) (2016), 179-202.

[17] K. Liu, P. Li, F. Han, W. Zhong, Global dynamics of nonlinear difference equation $x_{n+1}=A+x_{n} / x_{n-1} x_{n-2}$, J. Comput. Anal. Appl., 24(6) (2018), $1125-1132$.

[18] S. Moranjkić, Z. Nurkanović, Local and global dynamics of certain second-order rational difference equations containing quadratic terms, Adv. Dyn. Syst. Appl., 12(2) (2017), 123-157.

[19] M. Saleh, M. Aloqeili, On the rational difference equation $y_{n+1}=A+\frac{y_{n-k}}{y_{n}}$, Appl. Math. Comput. 171(1) (2005), 862-869.

[20] E. M. Elsayed, Dynamics of recursive sequence of order two, Kyungpook Math. J., 50 (2010), 483-497. 